ARTICLE

Received 2 Mar 2015 | Accepted 12 May 2015 | Published 30 Jun 2015

\title{
Early emergence of Yersinia pestis as a severe respiratory pathogen
}

Daniel L. Zimbler ${ }^{1}$, Jay A. Schroeder ${ }^{1}$, Justin L. Eddy ${ }^{1} \&$ Wyndham W. Lathem ${ }^{1}$

Yersinia pestis causes the fatal respiratory disease pneumonic plague. Y. pestis recently evolved from the gastrointestinal pathogen $Y$. pseudotuberculosis; however, it is not known at what point $Y$. pestis gained the ability to induce a fulminant pneumonia. Here we show that the acquisition of a single gene encoding the protease Pla was sufficient for the most ancestral, deeply rooted strains of $Y$. pestis to cause pneumonic plague, indicating that $Y$. pestis was primed to infect the lungs at a very early stage in its evolution. As $Y$. pestis further evolved, modern strains acquired a single amino-acid modification within Pla that optimizes protease activity. While this modification is unnecessary to cause pneumonic plague, the substitution is instead needed to efficiently induce the invasive infection associated with bubonic plague. These findings indicate that $Y$. pestis was capable of causing pneumonic plague before it evolved to optimally cause invasive infections in mammals.

\footnotetext{
${ }^{1}$ Department of Microbiology-Immunology, Northwestern University Feinberg School of Medicine, Chicago, Illinois 60611, USA. Correspondence and requests for materials should be addressed to W.W.L. (email: lathem@northwestern.edu).
} 
T he adaptation of bacterial pathogens to new hosts and specific microenvironments within the body typically occurs via the combined gain and loss of genetic elements during the evolution of the species. Y. pestis, the causative agent of plague ${ }^{1}$, represents an exceptional system for understanding how a pathogen adapts to new host environments to cause disease due to its recent emergence as a human pathogen and its relatively clonal nature $\mathrm{e}^{2-4}$. The divergence of $Y$. pestis from its progenitor species $Y$. pseudotuberculosis occurred within the last 10,000 years through multiple distinct genetic gains and losses, resulting in strikingly different modes of transmission and pathogenicity ${ }^{2,4,5}$. The soil- and water-borne enteropathogen $Y$. pseudotuberculosis causes the mild, self-limiting disease yersiniosis and is transmitted by the faecal-oral route ${ }^{1}$. In contrast, $Y$. pestis is transmitted by fleabites or aerosols, and causes the severely invasive and virulent disease plague ${ }^{1}$. Investigations on the genetic history of 118 annotated genomes of $Y$. pestis have revealed an evolutionary lineage that has defined both early ancestral (branch 0 ) and modern pandemic (branches 1 and 2) populations of $Y$. pestis ${ }^{6}$ based on sequential single-nucleotide polymorphism changes (Fig. 1a).

Indeed, recent work has begun to examine and retrace the specific genetic changes that were necessary for the transition from a mild self-limiting enteric disease to a severely invasive and virulent disease ${ }^{7-11}$. This change in disease and transmission is attributed to both the acquisition of new chromosomal and extrachromosomal DNA and as well as through genomic decay ${ }^{9,12}$. For example, the genes encoding for O-antigen synthesis on its lipopolysaccharide are inactivated in Y. pestis, resulting in a rough form of lipopolysaccharide ${ }^{13}$. Y. pestis also contains disruptions in the genes for the adhesins invasin and $\mathrm{YadA}^{14}$, both of which are functional in Y. pseudotuberculosis ${ }^{15,16}$. Furthermore, a recent study by Sun et al. ${ }^{7}$ has described only four minor genetic changes that were necessary for the flea-borne transmission of $Y$. pestis, which contributed directly to the ability of this pathogen to cause bubonic plague. In addition, the gain of the two plasmids pMT1 and PPCP1 that encode genes contributing directly to survival and virulence in both the flea and mammal is an apparent distinction of $Y$. pestis as it evolved from Y. pseudotuberculosis ${ }^{17}$.

$Y$. pestis can cause bubonic, septicemic and pneumonic plague in mammals. Of these, pneumonic plague is a unique respiratory syndrome that results in a purulent, multifocal, severe exudative bronchopneumonia and is the deadliest form of the disease, with fatality rates approaching $100 \%$ if untreated ${ }^{1,18}$. The disease is characterized as biphasic: the early phase is relatively asymptomatic and non-inflammatory, while the latter phase is highly pro-inflammatory, resulting in massive lobar lesions a

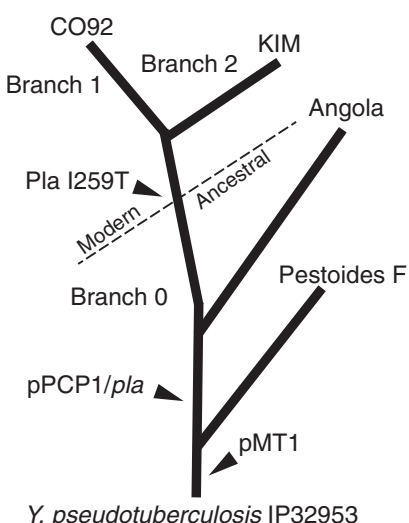

C

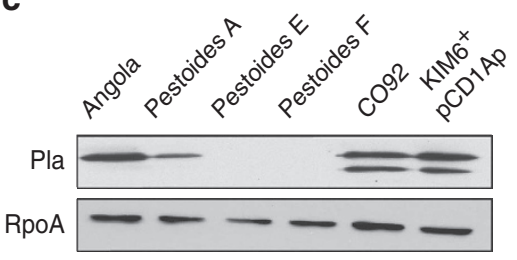

b
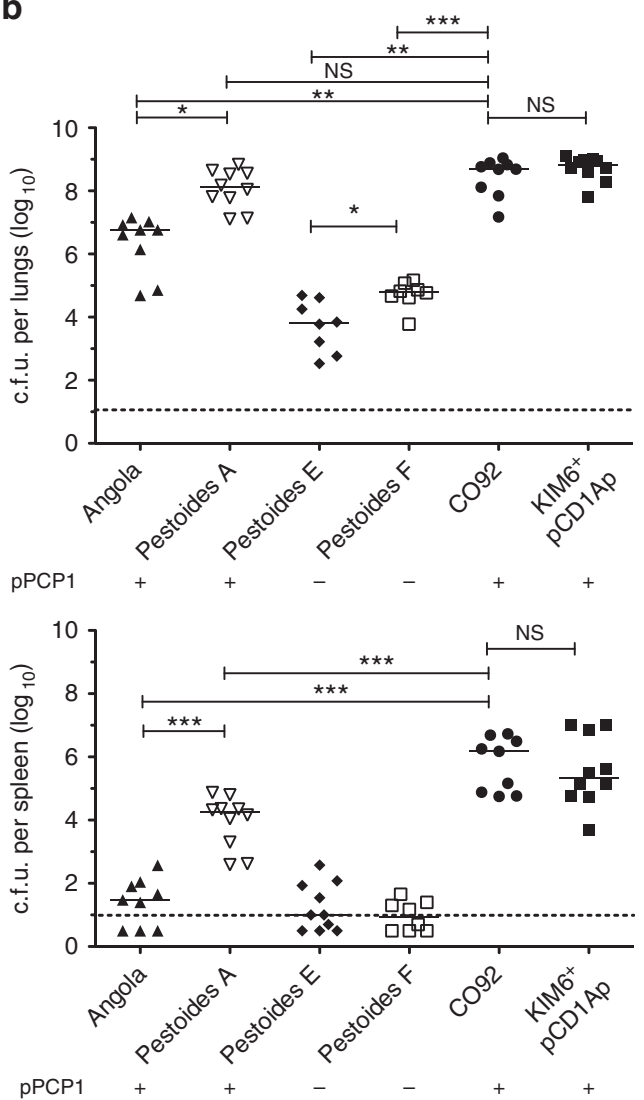

Figure 1 | pPCP1 is required by ancestral $\mathbf{Y}$, pestis to cause primary pneumonic plague. (a) Genomic maximum parsimony tree and divergence based on 16 Y. pestis genomes. The division between modern, pandemic strains and ancestral isolates is indicated. Tree was adapted from Morelli et al. ${ }^{5}$ (b) Bacterial burden within the lungs and spleens of mice $(n=10)$ infected i.n. with the indicated $Y$. pestis strains. Each point represents the numbers of bacteria recovered from a single mouse at $48 \mathrm{~h}$ post inoculation. The limit of detection is indicated by the dashed line and symbols in the dotted line indicate c.f.u. below the limit of detection. Symbols below the limit of detection represent mice that did not have detectable numbers of bacteria. A solid line indicates the median of c.f.u. recovered. The presence or absence of pPCP1 in each strain is indicated below. (c) Immunoblot analysis of whole-cell lysates of the indicated Y. pestis strains with antibodies to Pla and RpoA (as a loading control). The lower band represents the autoprocessed form of Pla (see Supplementary Fig. 5a). Full blots are shown in Supplementary Fig. 6. Panel is representative of three independent replicates. Data are combined from two independent experiments and error bars represent the s.e.m. $\left({ }^{\star} P \leq 0.05,{ }^{\star \star} P \leq 0.01,{ }^{\star \star \star} P \leq 0.001, N S\right.$, not significant by Mann-Whitney $U$-test). 
consisting primarily of infiltrating neutrophils, upregulation of cytokine and chemokine levels, and bacterial dissemination ${ }^{18}$.

One of the major virulence determinants encoded on the pPCP1 plasmid that is required for the development of pneumonic plague is the protease Pla. Similar in sequence and structure to OmpT of Escherichia coli and PgtE of Salmonella enterica, this $\beta$-barrel omptin family aspartic protease has been shown to have a wide range of proteolytic, adhesive and invasive properties necessary for $Y$. pestis pathogenesis in mammals ${ }^{19-21}$. While the most well-studied activity of Pla is the cleavage of host plasminogen (Plg) into the activated serine protease plasmin, to date only the host apoptotic molecule Fas ligand (FasL) has been defined in vivo as a substrate of Pla that contributes to primary pneumonic plague $22-24$. The amino-acid sequence of Pla is $100 \%$ conserved among all modern branched strains of $Y$. pestis; however, comparative genomics and substitutional analyses indicate that the sequence of Pla has shifted since the gene was introduced into ancestral branched strains of $Y$. pestis (Fig. 1a) ${ }^{8,25}$. In ancestral lineages such as Angola and Pestoides, Pla contains a single isoleucine substitution at position 259 (I259) located on the surface loop 5, whereas the modern lineages KIM and CO92 have a conserved threonine at this residue (T259) $26,27$. This single change has been shown in vitro to increase the efficiency of Plg activation and plasmin stability formed by the T259 variant of $\mathrm{Pla}^{28}$.

The acquisition of $\mathrm{pPCP} 1 / \mathrm{Pla}$ is positioned as an early occurrence in the emergence of pandemic strains of $Y$. pestis; however, there still exist ancestral isolates, such as Pestoides E and $F$ (isolated from voles in the Transcaucasian highland) ${ }^{29}$, that represent an intermediate lineage between Y. pseudotuberculosis and modern $Y$. pestis and do not carry PPCP1. However, these naturally occurring isolates of $Y$. pestis that do not harbour pPCP1 have nevertheless been reported to both colonize fleas and infect rodents ${ }^{30-32}$. Moreover, DNA of the pPCP1 plasmid, containing the modern T259 variant of Pla, has been detected in the remains of victims from both the Justinian plague and the Black Death, implying that most pandemic strains contained the genetic potential to be as virulent as strains currently associated with human disease $\mathrm{e}^{33,34}$.

This study was undertaken to identify and examine when during its emergence $Y$. pestis gained the ability to cause severe primary pneumonic plague. By utilizing the deeply rooted, ancestral Pestoides and Angola lineages, we demonstrate that the specific microevolutionary event of pPCP1/Pla acquisition contributed directly to the adaptation of $Y$. pestis to the respiratory environment, and no additional genetic changes were necessary to cause pneumonic plague. On the other hand, our results indicate that the T259 modification of the Pla protease enhanced systemic disease, suggesting that $Y$. pestis was primed to cause a fulminant pneumonia before its ability to efficiently cause invasive infections. Finally, we propose that the I259T substitution of Pla may have been one of the defining events that enabled pandemic spread of $Y$. pestis.

\section{Results}

Ancestral strains of $Y$. pestis rapidly outgrow in the lungs of mice. It is not known as to when during its emergence from the gastrointestinal pathogen $Y$. pseudotuberculosis that ancestral $Y$. pestis gained the ability to cause severe primary pneumonic plague. To address this question, we examined the capacity of the deeply rooted, ancestral $Y$. pestis strains Angola, Pestoides A, E and $\mathrm{F}$ (branch 0) (Fig. 1a), which display biochemical phenotypes and have environmental niches that are distinct from modern pandemic lineages and have not been associated with human disease $31,35,36$, to establish an infection in the lungs. We compared the bacterial outgrowth of these strains to that of the well-studied, modern-positioned lineage strains CO92 and KIM (branches 1 and 2) (Fig. 1a), both isolated from human plague cases, in an intranasal (i.n.) mouse model of pneumonic plague. We found that both Angola and Pestoides A, ancestral branched strains that carry pPCP1 (although with the I259 variant of Pla), are able to replicate to high levels in the lungs, approaching or equivalent to the bacterial burdens of the modern lineage strains CO92 and KIM (Fig. 1b). On the other hand, the Pestoides E and $\mathrm{F}$ strains are unable to rapidly proliferate in the pulmonary compartment (Fig. 1b), suggesting the absence of one or more virulence determinants required to cause primary pneumonic plague.

A comparison of these strains showed that a major distinguishing feature between Angola and Pestoides A versus Pestoides E and F is the presence of pPCP1, the plasmid encoding the virulence factor Pla; neither Pestoides E nor Pestoides $\mathrm{F}$ naturally carry pPCP1 (Supplementary Fig. 1a) ${ }^{29}$. Consistent with this, we found that all of the $\mathrm{pPCP}^{+}$strains are able to synthesize Pla to similar levels, however both Angola and Pestoides A do not autoprocess Pla due to the I259 variant of the protease carried by these strains ${ }^{28}$ (Fig. 1c). Thus, these data suggest that the gain of pPCP1 early during the evolution of $Y$. pestis was necessary for rapid bacterial outgrowth in the lungs.

Pestoides $F$ is unable to cause primary pneumonic plague. As Pestoides $\mathrm{F}$ is considered to be one of the most ancestral existing isolates of $Y$. pestis ${ }^{6,35}$ after it emerged from $Y$. pseudotuberculosis but before the acquisition of pPCP1, we further examined the interaction of this strain with the respiratory environment and in comparison with a mutant of CO92 lacking pla. To confirm that Pestoides F does not display functional activity generally attributed to Pla, we validated that Pestoides $\mathrm{F}$ is unable to activate Plg, similarly to an isogenic $\Delta$ pla mutant of CO92 $Y$. pestis (Supplementary Fig. 1b,c). We then infected mice via the i.n. route and followed survival over time; while all mice infected with the wild-type CO92 strain rapidly succumbed to the infection by day 4 post infection, the Pestoides F-infected mice showed a survival curve similar to that of $\Delta p l a$ CO92 and were significantly delayed in time to death compared with $\mathrm{CO} 92$ (Fig. 2a). To further assess the kinetics of infection of Pestoides $\mathrm{F}$ compared with CO92, we enumerated the colony-forming unit (c.f.u.) in the lungs and spleens at various times post infection and found that neither the $\Delta$ pla CO92 mutant nor the ancestral Pestoides $\mathrm{F}$ isolate are unable to replicate to high levels in the pulmonary compartment as compared with modern CO92 (Fig. 2b). Interestingly, by $48 \mathrm{~h}$ Pestoides $\mathrm{F}$ is able to modestly but significantly outgrow by $\sim 10-100$-fold in the lungs compared with mice infected with the $\Delta$ pla CO92 mutant, although similar numbers of bacteria are detected in spleens of Pestoides F and $\Delta$ pla CO92-infected mice at all time points examined (Fig. 2b).

To test the impact of Pestoides $\mathrm{F}$ on the host response during lung infection, we first examined the pathology of mouse lungs at $48 \mathrm{~h}$ post infection. Mice infected with wild-type CO92 produced large lobar pulmonary lesions throughout the lungs, whereas the lungs of Pestoides F-infected mice showed smaller, more nodular inflammatory lesions, similar in size and distribution to that of the $\Delta$ pla CO92 (Fig. 2c). We then performed flow cytometry to quantitate the observed influx of infiltrating cells into the lung airspace and to determine the distribution of cell types within the total cell population. CO92-infected mice showed a massive increase in the total number of cells recovered by bronchoalveolar lavage (BAL), and consistent with earlier reports ${ }^{37,38}$ the primary infiltrating cell type recruited to the lungs is the neutrophil 

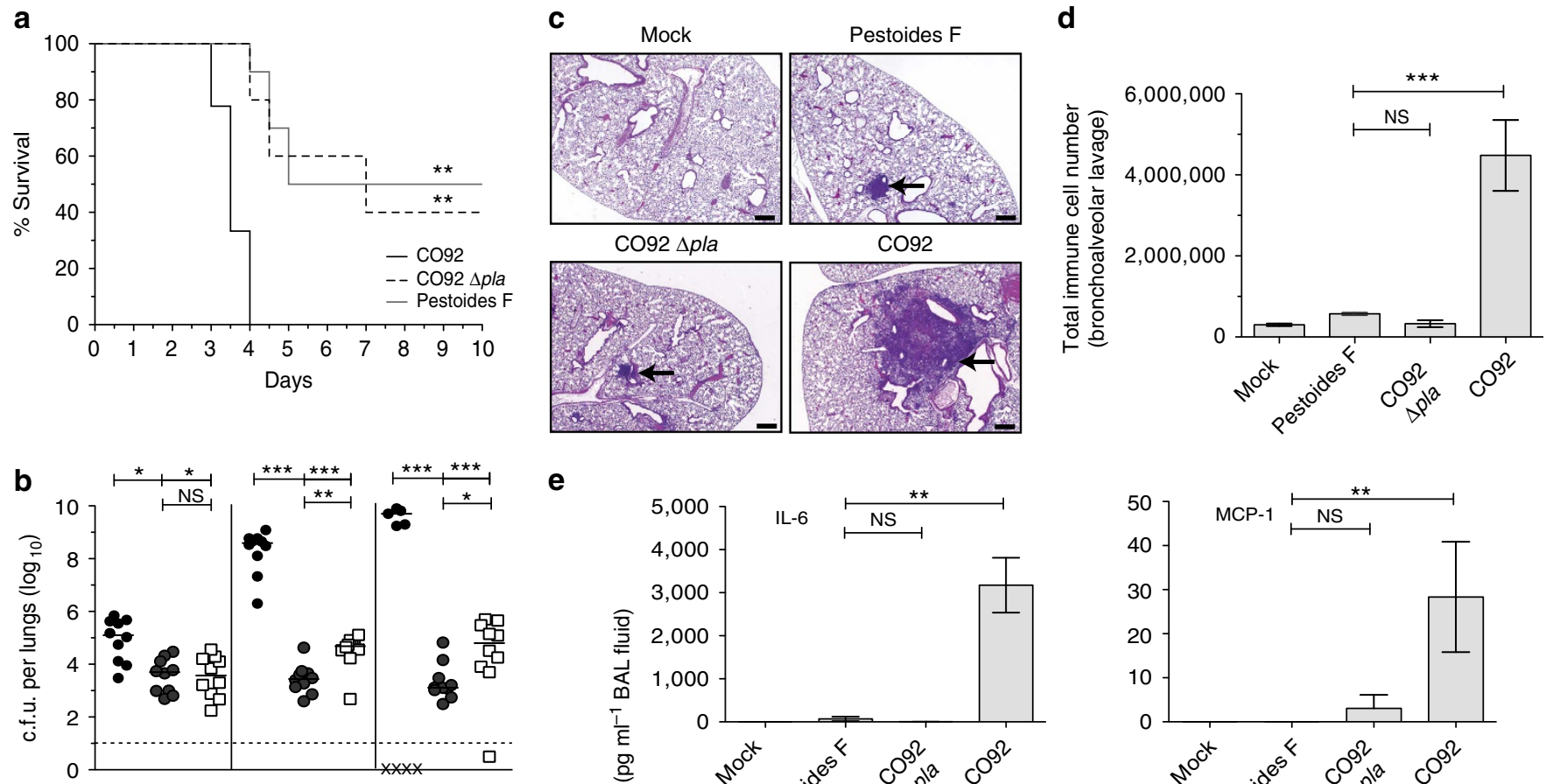

e
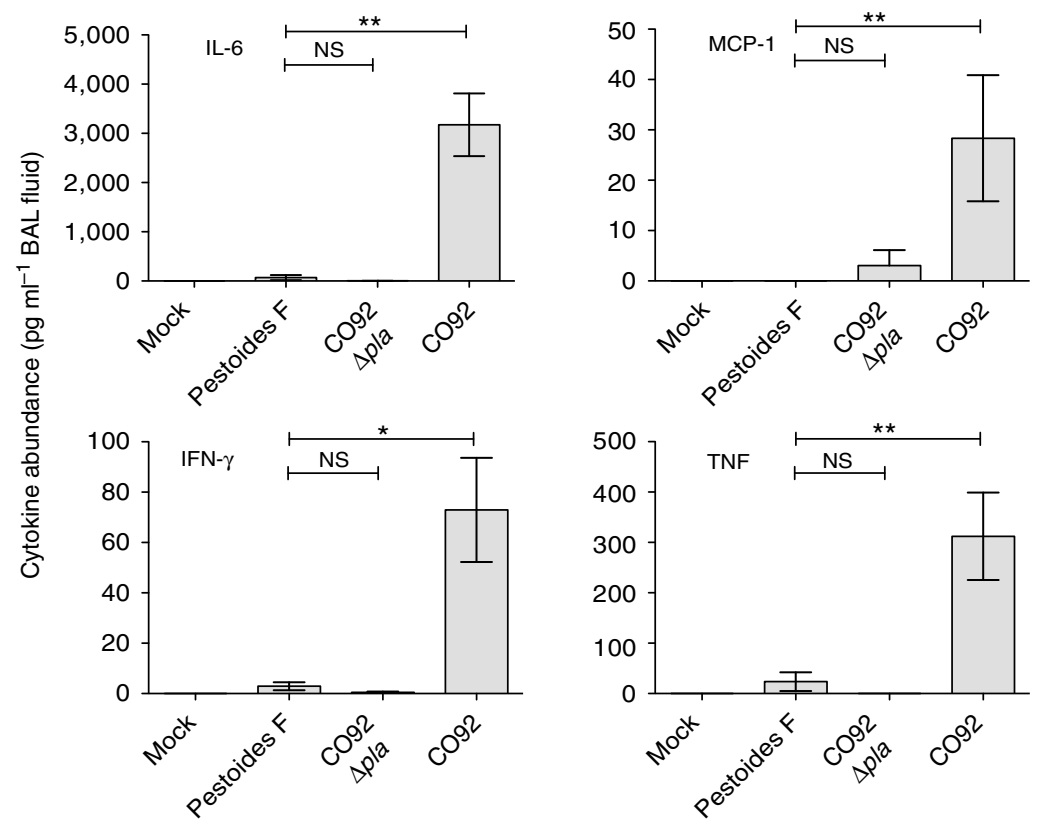

Figure 2 | Pestoides F resembles $\Delta$ pla Y. pestis during intranasal infections. (a) Survival of mice $(n=20)$ infected i.n. with Y. pestis CO92, CO92 $\Delta$ pla or Pestoides F. (b) Bacterial burden within the lungs and spleens of mice $(n=10)$ infected i.n. with the indicated Y. pestis strains, as described in Fig. 1. By $72 \mathrm{~h}$, mice begin to succumb to the CO92 infection (indicated by " $X$ " on the $x$ axis). (c) Pathology of mouse lung sections stained with $H \& E$ at $48 \mathrm{~h}$ post inoculation with PBS (mock), Pestoides F, CO92 $\Delta$ pla or CO92 Y. pestis. Representative images of inflammatory lesions are shown (arrows; $n=3$ ). Scale bar, $200 \mu \mathrm{m}$. (d) Enumeration of total immune cells present in BAL fluid $48 \mathrm{~h}$ post inoculation with PBS (mock), Pestoides F, CO92 $\Delta$ pla or CO92 Y. pestis $(n=10)$. (e) Abundance of the indicated inflammatory cytokines present in BAL fluid at $48 \mathrm{~h}$ post inoculation. Data are combined from two independent experiments and error bars represent the s.e.m. ( ${ }^{\star} P \leq 0.05$, ${ }^{\star} P \leq 0.01, N S$, not significant by Log-Rank test (survival), Mann-Whitney U-test (c.f.u.)). $\mathrm{H} \& \mathrm{E}$, haematoxylin/eosin.

(Fig. 2d; Supplementary Fig. 2). In contrast, mice infected with Pestoides F or the isogenic $\Delta$ pla CO92 mutant do not respond with this influx of immune cells to the lungs (Fig. 2d; Supplementary Fig. 2). Furthermore, an examination of the pulmonary inflammatory cytokine response reveals that, unlike CO92, which stimulates a robust release of multiple inflammatory cytokines into the airspace, mice infected with Pestoides $\mathrm{F}$ or the $\Delta$ pla CO92 mutant exhibit a severely dampened response with significantly lower cytokine levels (Fig. 2e). Thus, our data demonstrate that the ancestral Pestoides $\mathrm{F}$ strain is unable to cause primary pneumonic plague in a manner that is highly similar to a $\Delta$ pla mutant of CO92.

Pestoides F is competent to produce active Pla. On the basis of the similarities of the respiratory infection between Pestoides $\mathrm{F}$ and CO92 $\Delta$ pla, we asked whether the acquisition of pPCP1 (and specifically Pla) was sufficient for this ancestral $Y$. pestis strain to cause primary pneumonic plague. However, it is not yet known whether Pestoides $\mathrm{F}$ is able to stably harbour pPCP1 and produce active Pla. To test this, we introduced a kanamycin-marked pPCP1 derived from CO92 (ref. 14) into the $\mathrm{pCD}^{+}$and pCD1 ${ }^{-}$strains of Pestoides F. To ensure that this marked version of pPCP1 did not affect its encoded functions, we reintroduced the kanamycin-marked pPCP1 plasmid back into the $\mathrm{pCD} 1^{+}$ and $\mathrm{pCD}^{-}$strains of CO92 lacking pPCP1; in all cases, the kanamycin resistance marker was subsequently excised by Flpbased recombination. To test the ability of Pestoides F to carry pPCP1, we measured the copy number of the pla and pst genes (both encoded on pPCP1) in Pestoides F relative to that in CO92 and $\triangle$ pla CO92 carrying the reintroduced pPCP1. The copy number of pPCP1 carried by wild-type CO92 and the unmarked, reintroduced pPCP1 in CO92 are similar, indicating that the scar left by removal of the antibiotic cassette has no effect on plasmid replication (Fig. 3a). In addition, we found that Pestoides F naturally maintains pPCP1 without antibiotic selection (albeit at a higher relative copy number compared with CO92; Fig. 3a). 
Furthermore, neither Angola nor Pestoides A maintain pPCP1 at a significantly different copy number compared with wild-type CO92 (Supplementary Fig. 3a). To determine the conservation of pla regulation between ancestral and modern strains, a Ppla-gfp reporter containing the CO92 pla promoter cloned upstream of the coding sequence (CDS) for the green fluorescent protein (GFP) was integrated onto the chromosomes of CO92, Angola, Pestoides $\mathrm{A}$ and Pestoides $\mathrm{F}$ at the att $\operatorname{Tn} 7$ site. Bacteria were

a

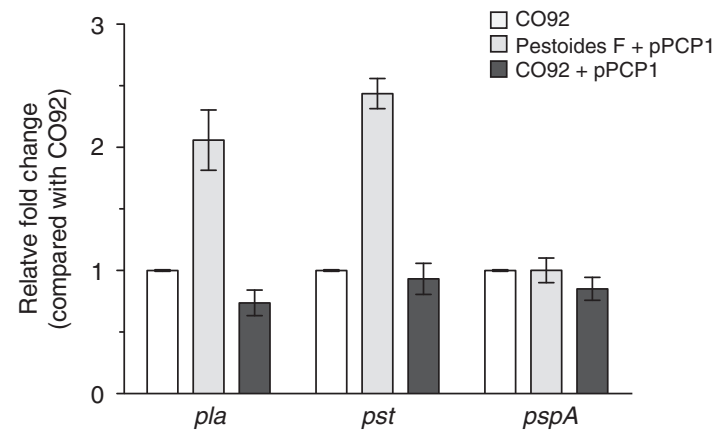

b

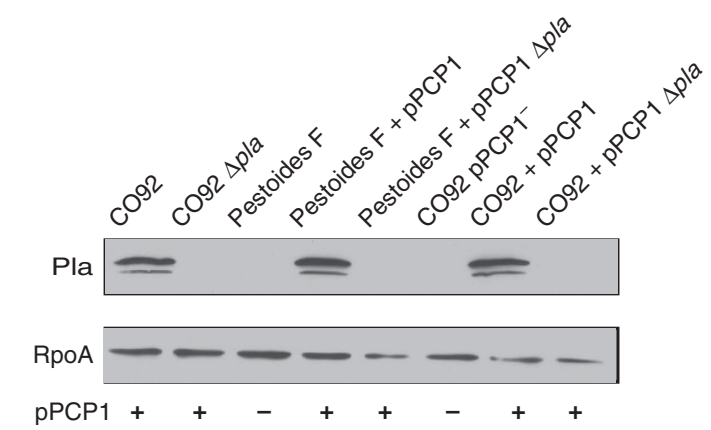

C

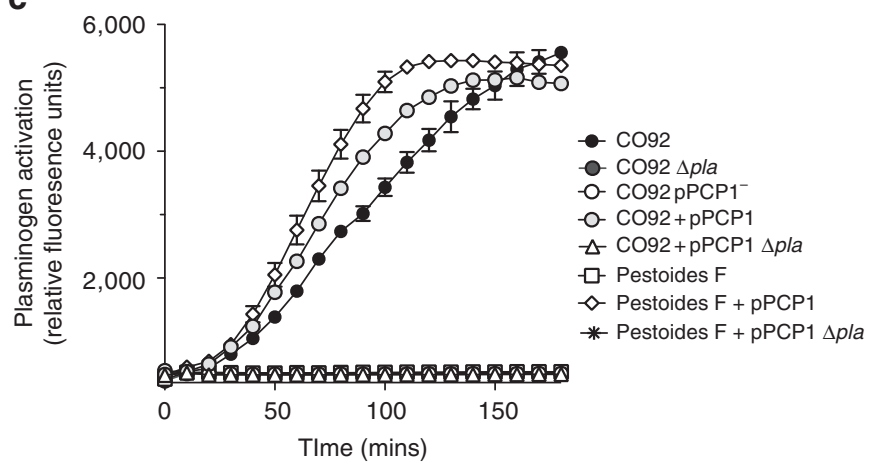

Figure 3 | Pestoides F is competent to carry pPCP1 and produce active Pla. (a) Relative copy number of pPCP1 (represented by the PPCP1-encoded genes pla and pst) in the pPCP1-reintroduced Pestoides F and CO92 strains, compared with $\mathrm{CO} 92$ (set at 1). pspA was used as a control for a chromosomal gene. Relative copy number for each gene was measured by quantitative PCR from gDNA isolated from cultures grown overnight at $37^{\circ} \mathrm{C}$ and normalized to gyrB. Data are combined from three independent biological replicates repeated twice; error bars represent the s.e.m.

(b) Immunoblot analysis of whole-cell lysates of indicated $Y$. pestis strains grown at $37^{\circ} \mathrm{C}$ with antibodies against Pla and RpoA (as a loading control). Panel is representative of three independent replicates. The presence or absence of pPCP1 in each strain is indicated. Full blots are shown in Supplementary Fig. 6. (c) The Plg-activating ability of the indicated Y. pestis $\mathrm{CO} 92$ or Pestoides $\mathrm{F}$ strains cultured at $37^{\circ} \mathrm{C}$ is shown. Data are representative of three independent experiments performed in triplicate; error bars represent the s.e.m. cultured at $37^{\circ} \mathrm{C}$ and the fluorescence of each strain was measured and normalized to the optical densities of the cultures. No significant change in fluorescence was observed between any of the ancestral strains compared with that of CO92 (Supplementary Fig. 3b), suggesting no differences in the regulation of pla transcription within these strains. Finally, immunoblot analysis of these strains cultured under the same conditions demonstrates that Pestoides F is able to synthesize Pla from pPCP1 at a similar level to that of CO92 (Fig. 3b).

To determine whether Pla properly localizes to the outer membrane and exhibits enzymatic activity when expressed in Pestoides F, we conducted a Plg activation assay and found that Pestoides F carrying pPCP1 showed similar levels of Plg activation compared with CO92 (Fig. 3c). Furthermore, additional Pla activity of Pestoides F was demonstrated by the similar efficiency as CO92 in the degradation of FasL (Supplementary Fig. 3c). The Pla activity of these Pestoides F or CO92 strains was subsequently eliminated in the isogenic $\Delta$ pla mutants (Fig. 3c; Supplementary Fig. 3c). Together, these data show that the ancestral strain Pestoides F can harbour pPCP1 and produce proteolytically active $\mathrm{Pla}$, indicating that additional changes to the $Y$. pestis genome were not necessary for the expression, production and activity of this essential virulence factor.

Pla is sufficient for Pestoides $\mathbf{F}$ to cause pneumonic plague. Pla is necessary for the progression of pneumonic plague in modern lineages of $Y$. pestis; therefore, we tested whether the acquisition of Pla by Pestoides $\mathrm{F}$ was all that was required for one of the earliest existing ancestral strains of $Y$. pestis to cause primary pneumonic plague. On introduction to mice via the i.n. route, we found that Pestoides F carrying pPCP1 was able to grow to high levels in the lungs and cause the death of the animals within 3-3.5 days, to the same extent and rate as wild-type Y. pestis CO92 (not statistically different, Mann-Whitney U-test; Fig. 4a,b). This increase in bacterial burden within the lungs is Pla dependent in both Pestoides F and CO92, as the time to death and enumerated c.f.u. were significantly decreased in the $\Delta p l a$ isogenic mutant infections in both backgrounds (Fig. 4a,b). An examination of the host response to Pestoides $\mathrm{F}$ harbouring pPCP1 revealed large lobar pulmonary lesions (Fig. 4c), a significant increase in total cell number (Fig. 4d) with neutrophils being the primary infiltrating cell type (Supplementary Fig. 4a), and a robust inflammatory cytokine response, all which are similar to that caused by modern Y. pestis strain CO92 (Supplementary Fig. 4b). In total, our results demonstrate that the acquisition pPCP1/Pla by one of the most deeply rooted strains of $Y$. pestis was sufficient for this newly emerged species to cause primary pneumonic plague within rodents.

Pla I259T modification was not required to cause pneumonic plague. A single residue substitution (I259T) in Pla between ancestral and modern lineages of $Y$. pestis (Fig. 1a) indicates that Pla has undergone natural adaptation since its acquisition by Yersinia. Therefore, we asked whether this modification was required by or enhanced the ability of $Y$. pestis to cause primary pneumonic plague. To do so, we introduced the I259 amino-acid substitution into Pestoides F + pPCP1 as well as into the modern CO92 isolate, thus generating the ancestral variant of Pla within both ancestral and modern Y. pestis strains. Immunoblot analysis of these strains confirms that the I259 variant of Pla does not autoprocess itself and has a reduced rate of Plg activation compared with the T259 variant in both modern and ancestral $Y$. pestis, consistent with a previous study on the I259 variant ${ }^{28}$ (Supplementary Fig. 5a,b). However, the I259 Pla variant is still able to degrade FasL to the same efficiency as Pla T259 
a

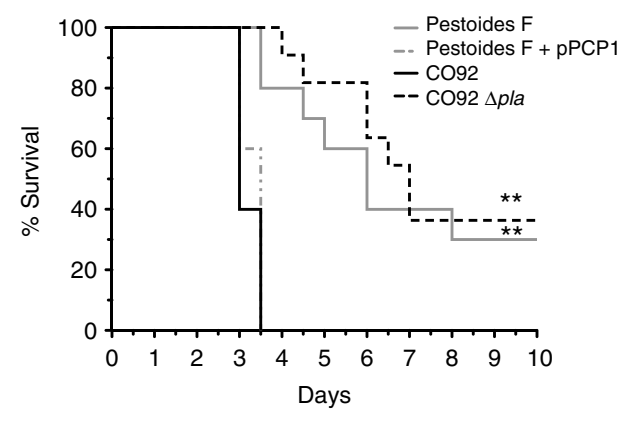

b

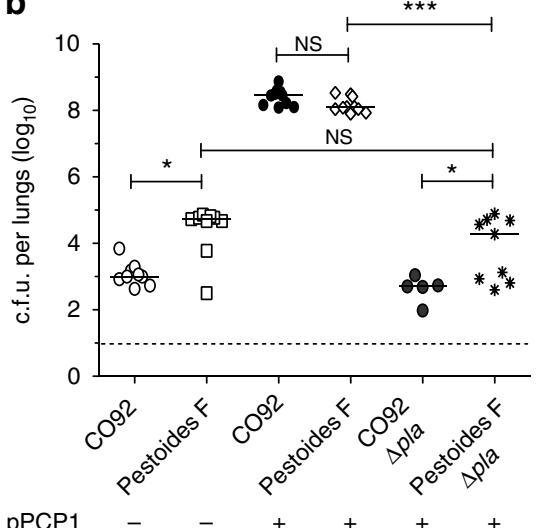

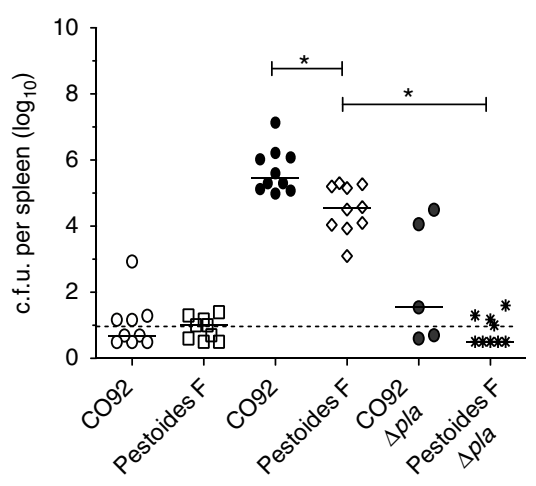

pPCP1 - $\quad+\quad+\quad+\quad+\quad+$ c

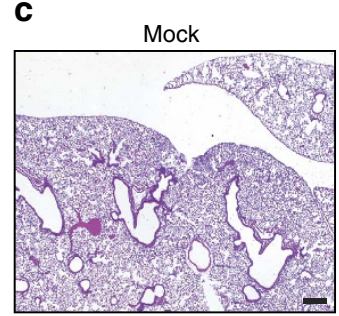

Pestoides $\mathrm{F}$

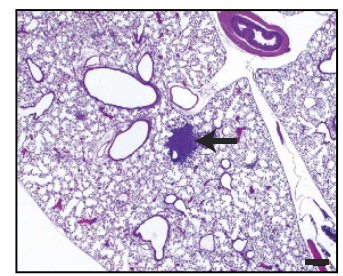

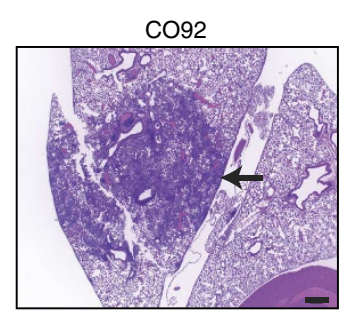

Pestoides F + pPCP1

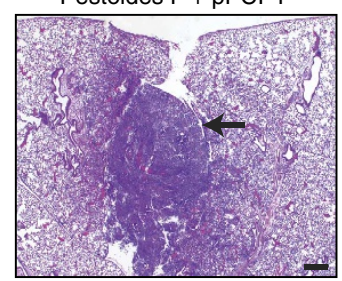

C092 spla

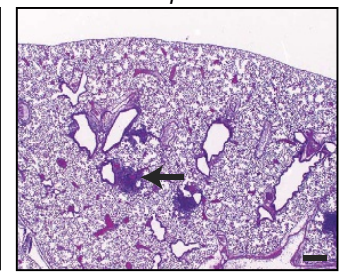

Pestoides F + pPCP1 1 pla

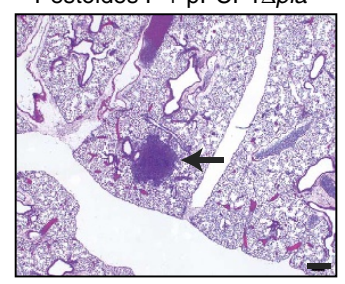

d

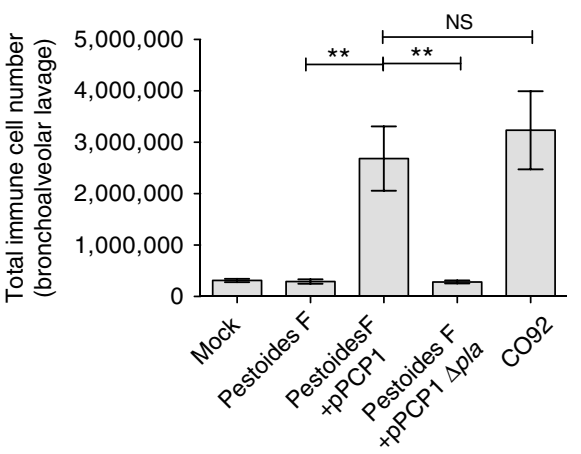

Figure 4 | The acquisition of Pla by Pestoides $\mathbf{F}$ is sufficient to cause primary pneumonic plague. (a) Survival of mice $(n=10)$ infected i.n. with the indicated strains of $Y$. pestis. (b) Bacterial burden within the lungs and spleens of mice $(n=10)$ infected i.n. with the indicated Pestoides $F$ or CO92 strains, as described in Fig. 1. The presence or absence of pPCP1 is indicated. (c) Pathology of mouse lung sections stained with H\&E at $48 \mathrm{~h}$ post inoculation with PBS (mock) or the indicated Y. pestis strains. Representative images of inflammatory lesions (arrows; $n=3$ ) are shown. Scale bar, $200 \mu \mathrm{m}$. (d) Enumeration of total immune cells present in BAL fluid $48 \mathrm{~h}$ post inoculation with PBS (mock) or the indicated Y. pestis strains ( $n=10$ ). Data are combined from two independent experiments. Error bars represent the s.e.m. $\left({ }^{\star} P \leq 0.05,{ }^{\star \star} P \leq 0.01,{ }^{\star \star \star} P \leq 0.001, N S\right.$, not significant; Log-Rank test (survival), Mann-Whitney U-test (c.f.u.)). H\&E, haematoxylin/eosin.

(Supplementary Fig. 5c,d). We also examined the rate of Plg activation by Pestoides A (which expresses the I259 variant of Pla), and observed that this isolate has reduced activity even compared with other strains expressing the same I259 variant of Pla (Supplementary Fig. 5b).

We next asked whether the T259 variant of Pla enhances virulence during primary pneumonic plague compared with the Pla I259 in either the modern (CO92) or ancestral (Pestoides F) strains of $Y$. pestis by assessing the bacterial burden in the lungs after $48 \mathrm{~h}$. We found no significant difference (Mann-Whitney $U$-test) in c.f.u. in the lungs of Pestoides F or CO92 expressing the I259 variant of Pla compared with the same strains expressing Pla T259 (Fig. 5a), indicating that this single amino-acid substitution was not required for $Y$. pestis to infect the lungs and rapidly replicate. However, there were significantly fewer bacteria in the spleens of mice infected with Pla I259 compared with the T259 variant in the Pestoides $\mathrm{F}$ isolate (Fig. 5a). Together with the data presented in Fig. 4, our results indicate that once Y. pestis acquired $\mathrm{Pla}$, no additional genetic changes were required for the plague bacillus to cause a rapidly progressing pneumonic infection; rather this modification may instead be involved in dissemination from the lungs and/or bacterial survival during systemic infection.
Pla I259T modification was necessary for invasive infections. As we observed reduced numbers of bacteria in the spleens following i.n. infection with Pestoides F + Pla I259 compared with Pla T259, the data shown in Fig. 5a led us to hypothesize that this modification of Pla may be more significant for dissemination or survival of $Y$. pestis during systemic, invasive infections, such as occurs during bubonic plague. To test this, we used the same strains in a subcutaneous (s.c.) mouse model of infection of bubonic plague and measured bacterial burden within the inguinal lymph nodes and spleens of infected mice. Mice infected with either the ancestral or modern Pla variant in the Pestoides F or CO92 background resulted in equivalent bacterial outgrowth within the inguinal lymph node after 3 days (Fig. 5b). However, we detected $\sim 100$-fold fewer bacteria in the spleens of mice infected with the ancestral I259 variant of Pla compared with Pla T259 in both the ancestral and modern strains of Y. pestis (Fig. 5b). Moreover, Pestoides F is able to outgrow in the lymph node; however, it only disseminates to distal sites by day 3 when harbouring pPCP1 (Fig. 5b). Thus, these observations indicate that the I259T modification of Pla was important for $Y$. pestis to cause a disseminated, invasive infection from the site of inoculation, such as occurs during bubonic plague, in both modern and ancestral strains of $Y$. pestis. 
a
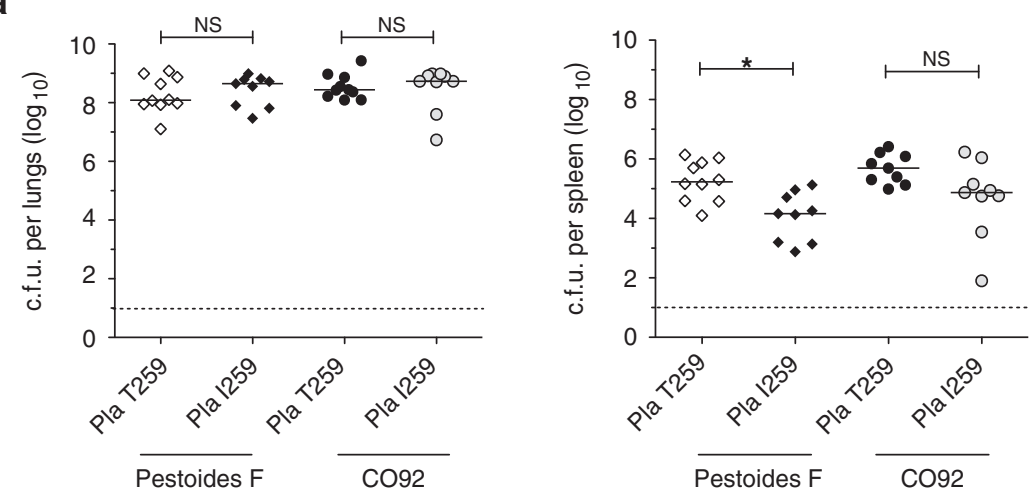

b
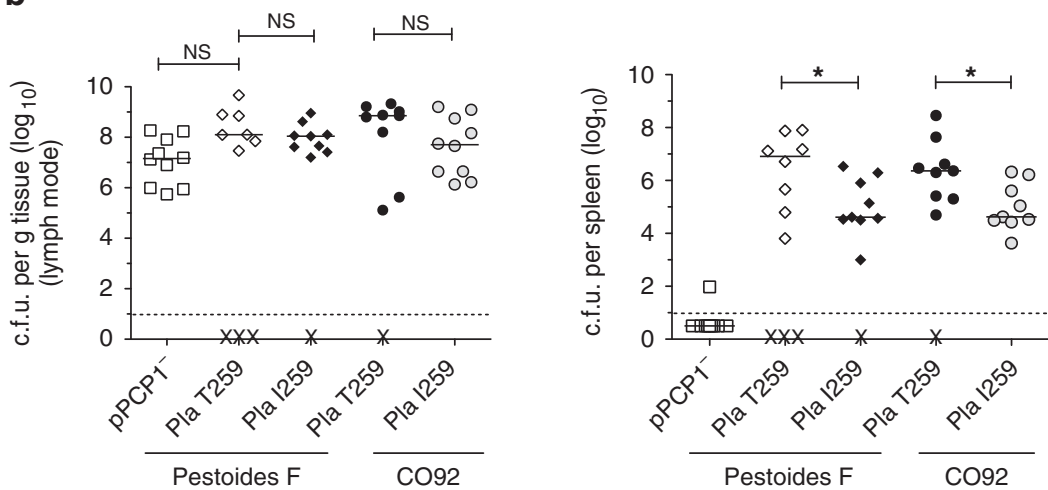

Figure 5 | The ancestral variant of Pla is sufficient to cause pneumonic plague but not optimal systemic infection. (a) Bacterial burden within the lungs and spleens of mice $(n=10)$ infected i.n. with Y. pestis CO92 or Pestoides F carrying PPCP1 with either the T259 or 1259 variant of Pla, as described in Fig. 1. (b) Bacterial burden within the inguinal lymph nodes and spleens of s.c. infected mice $(n=10)$ with wild-type Pestoides F, or CO92 or Pestoides F carrying pPCP1 with either the T259 or 1259 variant of Pla. The variant of Pla in each strain is indicated. Data are combined from two independent experiments and error bars represent the s.e.m. ( ${ }^{\star} P \leq 0.05$, NS, not significant by Mann-Whitney U-test).

\section{Discussion}

The mechanisms by which new bacterial pathogens emerge are not well understood, particularly in regards to the specific gain or loss of individual genetic determinants that enable the adaptation and development of acute disease in discrete hosts and/or microenvironments. These genetic changes are believed to account for phenotypic distinctions and pathogenic potential observed between lineages of $Y$. pestis, as genetic diversity is a known source of phenotypic diversity underlying disease dynamics. ${ }^{39,40}$ However, the molecular mechanisms that $Y$. pestis acquired to specifically become a severe respiratory pathogen are not yet established, despite several comparative genomic, transcriptomic and proteomic studies $6,8,41$.

Before this study, it was unknown whether ancestral strains of $Y$. pestis were capable of causing primary pneumonic plague and at what point during the evolution of $Y$. pestis they became competent to do so. Here we report that ancestral, branch 0 strains of $Y$. pestis gained the ability to successfully replicate to high levels within the pulmonary compartment very early in their evolution from Y. pseudotuberculosis, but only if they had acquired the gene encoding the Pla protease. This provides additional support for the acquisition of pPCP1 as a critical step in the adaptation of $Y$. pestis to the pulmonary environment. While it has been demonstrated that $Y$. pseudotuberculosis can colonize the lungs and stimulate a pneumonia in mice, the disease is delayed and does not exhibit the pulmonary pathology or rapid systemic spread that is observed during infections with Y. pestis ${ }^{42}$. Furthermore, $Y$. pseudotuberculosis is not known to naturally cause epidemics of respiratory disease.
While Pestoides $\mathrm{F}$ is one of the earliest existing $Y$. pestis strains in branch 0 following the divergence of the species from Y. pseudotuberculosis (Fig. 1a), here the addition of pPCP1 now confers Pestoides F with the higher pathogenicity observed in respiratory infections that are exhibited by the modern CO92 and KIM strains. This is the only genetic change needed by one of the most deeply rooted of all $Y$. pestis strains to cause a fulminant lung infection, and indicates that even during the transitional evolutionary state between $Y$. pseudotuberculosis and modern $Y$. pestis, this ancestral species was primed to cause pneumonic plague. In contrast, the addition of $\mathrm{pPCP} 1$ to $Y$. pseudotuberculosis does not result in proteolytically active Pla or enhance virulence in mice, as Pla produced by $Y$. pseudotuberculosis is sterically inhibited by the presence of $\mathrm{O}$-antigen on the bacterial surface $24,43,44$. While the pPCP1 plasmid itself is not sufficient to explain all of the differences in virulence between $Y$. pseudotuberculosis and Y. pestis, our data does indicate that ancestral strains could initiate epidemics of pneumonic plague as soon as pPCP1 was acquired.

The origin of the pPCP1 plasmid remains unknown, although it encodes a ColE1-like origin of replication, suggesting a common ancestry with plasmids typically found in enteric organisms ${ }^{31}$. The plasmid encodes three proteins; thus far Pla is the only virulence determinant encoded on PPCP1 shown to be important for development of enhanced disease by modern $Y$. pestis ${ }^{37,45}$, a conclusion that is further supported by this work. In addition, our data clearly indicate that not only the acquisition but also the modification of Pla was a crucial adaptation in the systemic spread of $Y$. pestis from both the lungs and the lymph 
node. The prominent function of Pla is thought to be the proteolytic activation of host Plg into plasmin, which allows the bacteria to escape from immobilizing fibrin clots and potentiates fibrinolysis. During infection with $Y$. pestis carrying the I259 variant of Pla, colonization and outgrowth within the lungs are unaffected; however, dissemination is significantly reduced compared with the T259 variant, likely due to reduced Plg activation and stable plasmin formation by Pla during infection. During pneumonic plague, mortality is due to the pneumonia developed within the lungs, whereas during bubonic plague the animal dies from sepsis due to dissemination of bacteria from the lymph node, and not from localized lymphadenopathy ${ }^{17,18,22}$. As our data show that the I259T substitution significantly enhanced the invasive capacity of $Y$. pestis during bubonic plague, it is tempting to speculate that this modification of Pla may have contributed to the 'big bang' event, recently proposed by Cui et al. ${ }^{6}$, that resulted in the transition from localized outbreaks of plague to the pandemic spread of $Y$. pestis.

This study expands on previous work by Welkos et al. ${ }^{46}$ and Worsham and Roy ${ }^{30}$, which showed that Pestoides $\mathrm{F}$ has a comparable $\mathrm{LD}_{50}$ (dose lethal to $50 \%$ ) to $\mathrm{CO} 22$ in both the aerosol and s.c. routes of infection. Data presented here suggest that the outcomes of disease observed in these earlier reports are likely due to bacteremia following escape of Pestoides $\mathrm{F}$ from the lungs or via direct inoculation into the blood following s.c. or intradermal injection (such as observed with $Y$. pestis CO92 $\Delta$ pla), ${ }^{17,37}$ rather than the severe exudative bronchopneumonia or bubonic infection that occurs with $\mathrm{pPCP}^{+}$ strains. Indeed, $\mathrm{Pla}$ is dispensable once $Y$. pestis enters the blood $^{45}$, suggesting that $\mathrm{pPCP} 1^{-}$strains such as Pestoides E and $\mathrm{F}$ may be maintained in the environment at low levels through direct bloodstream infections via flea or animal bite. Furthermore, in light of these previous studies, our data suggest that the acquisition of Pla enabled new, more rapidly progressing forms of disease caused by $Y$. pestis, rather than increased lethality.

The ability of Pestoides $\mathrm{F}$ to modestly outgrow in the pulmonary compartment compared with $\Delta$ pla CO92 supports its placement as a transitional lineage of $Y$. pestis; however, it is unable to cause pneumonic plague. This observation can best be explained by Pestoides F maintaining specific variations in metabolic pathways that allow for increased fitness within the host. Comparative genomics and transcriptomics have revealed frameshifts, point mutations and pseudogenes, which may account for phenotypic distinction in nutritional requirements, carbohydrate fermentation and other biochemical properties between $\mathrm{CO} 92$ and Pestoides $\mathrm{F}^{35,39,41}$. For instance, the ability to catabolize L-aspartate into fumarate by aspartase (AspA) has been lost by modern Y. pestis lineages such as CO92 and KIM, whereas Y. pseudotuberculosis and the ancestral Angola and Pestoides isolates of $Y$. pestis maintain AspA activity ${ }^{47}$. On the other hand, although the Angola isolate encodes pla, this strain is not able to grow to the same levels in the lungs as modern isolates, suggesting that Angola may have diverged from the branch 0 group in a way that has ultimately reduced its fitness in the pulmonary compartment. It is of interest to determine the specific genetic differences between Angola, the Pestoides and modern Y. pestis that could account for these variations in virulence.

Retracing the evolution of $Y$. pestis since its emergence has revealed multiple small genetic changes that have altered host tropism, transmission and virulence ${ }^{12,48}$. For example, investigations into the adaptation of $Y$. pestis to flea-borne transmission have uncovered only a few genetic alterations that facilitated this ability $7,11,49,50$. The increasing genomic comparisons of global Y. pestis isolates have revealed that it has evolved clonally with little genetic diversity; however, it is becoming apparent that these small genetic mutations resulting in the change of its transmission have been strongly positively selected for during its evolution. The emergence of new pathogenic clones has been directly linked with both the gain and loss of genetic elements by bacteria that were previously thought to be non-virulent ${ }^{51}$. Moreover, single gene acquisition events have been demonstrated to have fundamental effects on an organism's evolutionary adaptation to new host ${ }^{52-54}$. Indeed, our data suggest that the emergence of other new respiratory pathogens could occur similarly via relatively small gene acquisition events. Taken together, our results indicate that $Y$. pestis was primed to cause an acute respiratory disease early on during its evolution from Y. pseudotuberculosis and that this occurred before $Y$. pestis was able to develop a fully optimized invasive infection.

\section{Materials and methods}

Reagents, bacterial strains and culture conditions. All reagents used in this work were purchased from Sigma-Aldrich or VWR unless otherwise stated. The bacterial strains used in this work are listed in Supplementary Table 1, plasmids are listed in Supplementary Table 2 and oligonucleotide sequences are given in Supplementary Table 3. Brain-heart infusion (BHI) broth or agar (Difco) was used to maintain $Y$. pestis strains and derivatives. Luria-Bertani broth or agar was used to maintain all E. coli strains. Ampicillin $\left(100 \mu \mathrm{g} \mathrm{ml}^{-1}\right)$ or kanamycin

$\left(50 \mu \mathrm{g} \mathrm{ml}^{-1}\right)$ was added to the medium as needed. For animal infections, Y. pestis strains were cultured in $10 \mathrm{ml}$ BHI in $125 \mathrm{ml}$ Erlenmeyer flasks with shaking at 250 r.p.m. at either $37^{\circ} \mathrm{C}$ with the addition of $2.5 \mathrm{mM} \mathrm{CaCl}_{2}$ for i.n. infections or $26^{\circ} \mathrm{C}$ for s.c. infections ${ }^{37,55}$. All experiments using select agent strains of $Y$. pestis were conducted in an approved BSL-3/ABSL-3 facility at Northwestern University.

Mutagenesis and generation of $\boldsymbol{Y}$. pestis strains. Recombinant DNA procedures were approved by the Institutional Biosafety Committee of Northwestern University. pCD1 ${ }^{+}$was cured from $Y$. pestis strains by passage on BHI plates containing magnesium oxalate and incubation at $37^{\circ} \mathrm{C}^{56}$. The loss of pCD1 in these strains was further confirmed by PCR and plasmid profile analysis ${ }^{57}$. Y. pestis $\mathrm{KIM}^{+}$was transformed with pCD1Ap by electroporation. Transformants were selected on $\mathrm{BHI}+$ ampicillin + Congo Red following growth at $26^{\circ} \mathrm{C}$ for 2 days, and transformants were additionally screened for their ability to grow on BHI at $26^{\circ} \mathrm{C}$ but were unable to grow on BHI plates containing magnesium oxalate at $37^{\circ} \mathrm{C}$. The presence of pCD1Ap was further confirmed by PCR.

The kanamycin-marked version pPCP1 derived from CO92 was electroporated into $\mathrm{pCD} 1^{+}$or $\mathrm{pCD}^{-}$strains of Pestoides F. To ensure that this variant of pPCP1 remained isogenic with CO92 and did not impact functions associated with pPCP1 (for example, Pla activity), we reintroduced the kanamycin-marked pPCP1 plasmid back into the $\mathrm{pCD}^{+}{ }^{+}$and $\mathrm{pCD}^{-}$strains of CO92 lacking pPCP1. Tranformants were selected on $\mathrm{BHI}+$ kanamycin following growth at $26^{\circ} \mathrm{C}$ for 2 days. The kanamycin resistance cassette used for the selection of transformants was excised using Flp recombinase methods by electroporation of pSkippy as described previously ${ }^{58}$. The presence of unmarked pPCP1 in each strain was confirmed by PCR and plasmid profile analysis ${ }^{57}$. Unmarked isogenic derivatives of pla were generated in the $\mathrm{pCD}^{+}$or $\mathrm{pCD}^{-}$strains introduced with $\mathrm{pPCP} 1$ using a modified form of lambda red recombination ${ }^{37}$, and the kanamycin resistance cassette excised as above. The ancestral Pla variant (I259) in the CO92 and Pestoides F strains were generated by the PCR-based method of overlap extension before introduction into the respective strain carrying pWL204 (ref. 37).

Plg activation assay, cleavage of FasL and immunoblot analyses. The Plg-activating ability of $Y$. pestis strains was assessed ${ }^{37,59}$. Briefly, strains were cultured for $6 \mathrm{~h}$ at $37^{\circ} \mathrm{C}$ before being diluted to $4 \times 10^{6}$ c.f.u. in $20 \mathrm{mM}$ HEPES buffer and combined with purified human glu-Plg (Haematologic Technologies; $4 \mu \mathrm{g})$ and the chromogenic substrate D-AFK-ANSNH- $\mathrm{iC}_{4} \mathrm{H}_{9}-2 \mathrm{HBr}(\mathrm{SN} 5$; Haematologic Technologies; $50 \mu \mathrm{M}$ ) in a total volume of $200 \mu \mathrm{l} 20 \mathrm{mM}$ HEPES buffer and incubated at $37^{\circ} \mathrm{C}$ for $3 \mathrm{~h}$. The absorbance at $460 \mathrm{~nm}$ was measured every 10-11 min in a Molecular Devices SpectraMax M5 fluorescence microplate reader. Data are representative of at least three independent experiments.

The cleavage of the Pla substrate FasL by $Y$. pestis strains was assessed as described previously ${ }^{38}$. Briefly, strains were cultured for $6 \mathrm{~h}$ at $37^{\circ} \mathrm{C}$ before being diluted to $4 \times 10^{6}$ c.f.u. in $20 \mathrm{mM}$ HEPES and combined with recombinant HA-tagged mouse FasL (R\&D systems; $0.1 \mu \mathrm{g}$ ) and incubated at $37^{\circ} \mathrm{C}$. Following incubation for $10,15,30$ or $60 \mathrm{~min}$, bacteria were removed by centrifugation and proteins contained within the supernatant were mixed with reducing sample buffer, separated by SDS-polyacrylamide gel electrophoresis and transferred to nitrocellulose for analysis by immunoblotting with an anti-HA antibody (1:10,000 dilution; Roche, clone 3F10).

To examine Pla production, bacteria were cultured for $6 \mathrm{~h}$ at $37^{\circ} \mathrm{C}$ and one optical density unit (equivalent to $\sim 1 \times 10^{9}$ c.f.u.) of each culture was harvested 
and mixed with reducing sample buffer, separated by SDS-polyacrylamide gel electrophoresis and transferred to nitrocellulose for analysis by immunoblotting with antibodies to Pla (1:200 dilution) or RpoA as a loading control (1:100,000 dilution; Melanie Marketon, Indiana University), as described previously ${ }^{60}$.

Plasmid copy number determination. The relative copy number of pPCP1 maintained by $Y$. pestis strains was determined as described ${ }^{61}$. Briefly, genomic DNA (gDNA) was isolated using the Wizard gDNA isolation kit (Promega) from bacteria cultured at $37^{\circ} \mathrm{C}$ according to the manufacturer's instructions. Quantitative PCR was performed on gDNA with primers specific for $g y r B$, $p s p A$, $p s t$ and pla (Supplementary Table 2). Relative plasmid copy number was determined by the formula $2^{-\Delta \mathrm{CT}}$, where $\Delta \mathrm{CT}$ is the threshold cycle difference between $\operatorname{gyr} B$ and the gene of interest. Statistical differences were determined by the Student's $t$-test from three independent experiments.

GFP assays. To assess pla expression in Y. pestis strains, a reporter construct containing 500 base pairs upstream of the pla CDS was fused to the CDS of GFP $\left(\mathrm{P}_{\text {pla }}{ }^{-\mathrm{gfp}}\right)$ and integrated onto the chromosome of the indicated Y. pestis $\left(\mathrm{pCD1}^{-}\right)$ strains at the att $\operatorname{Tn} 7$ site contained within the glmS-gstS intergenic region via the Tn7 site-specific transposon ${ }^{58,60,62}$. Integrations were confirmed by PCR. Strains carrying the $\mathrm{P}_{\text {pla }}$-gfp reporter were assessed for relative fluorescence at $37^{\circ} \mathrm{C}$ in triplicate as described previously using a Tecan Safire microplate reader ${ }^{58}$. Background fluorescence subtraction and normalization were performed as described ${ }^{58}$. Statistical differences were determined by the Student's $t$-test from three independent experiments.

Animal infections. All procedures involving animals were carried out in compliance with protocols approved by the Institutional Animal Care and Use Committee of Northwestern University. C57BL/6 female mice, aged 6-8 weeks, were obtained from The Jackson Laboratories (Bar Harbor, ME). Mice were lightly anaesthetized with ketamine and xylazine and infected by the i.n. route $\left(10^{4}\right.$ c.f.u. in $4 \times 5 \mu \mathrm{l}$ aliquots) or the s.c. route (injection of 50 c.f.u. in $100 \mu \mathrm{l}$ with a 28 gauge needle at the left inner thigh) with Y. pestis strains diluted in PBS. The inoculating dose was confirmed by plating on BHI agar following incubation at $26^{\circ} \mathrm{C}$ for 2 days. Mice were monitored twice daily for the length of the experiment. Mice were euthanized by i.p. injection of pentobarbital sodium. All animal infections were performed at least twice and the data combined.

To determine bacterial burden, mice were inoculated i.n. or s.c. with Y. pestis strains and euthanized at various times post infection. For i.n. infections, the lungs and spleens were removed, and for s.c. infections, the left inguinal lymph nodes and spleens were removed. Organs were subsequently weighed, homogenized in sterile PBS, serially diluted and plated onto BHI agar. Following incubation at $26^{\circ} \mathrm{C}$ for 2-3 days, the c.f.u. per organ was enumerated. To determine survival of mice following infection, mice were inoculated i.n. with $Y$. pestis and derivatives and monitored every $12 \mathrm{~h}$ for up to 10 days. Statistical significance was calculated by the Kaplan-Meier test for survival experiments and a two-tailed Mann-Whitney $U$-test for bacterial burden measurements.

Histopathology. Mice were inoculated i.n with Y. pestis strains, and at $48 \mathrm{~h}$ post infection mice were euthanized and lungs inflated with $1 \mathrm{ml}$ of $10 \%$ neutral buffered formalin via tracheal cannulation. Lungs were removed, fixed in $10 \%$ formalin and subsequently embedded in paraffin. Two $5-\mu \mathrm{m}$ sections, $200 \mu \mathrm{m}$ apart per lung, were stained with haematoxylin/eosin (H\&E) for examination. Tissue embedding, sectioning and staining with haematoxylin/eosin were performed by the Northwestern University Mouse Histology and Phenotyping Laboratory. Slides were imaged using a Zeiss Axioskop/Nuance Camera.

Innate immune cell quantification. Mice were infected i.n. with $Y$. pestis strains or mock-infected with PBS, and at $48 \mathrm{~h}$ post infection mice were euthanized and BAL was performed ${ }^{38}$ using $1 \mathrm{ml}$ PBS for each lavage for a total of five lavages per animal. Samples were centrifuged at $300 \mathrm{~g}$ for $10 \mathrm{~min}$ to separate cells and cell debris; the supernatant from the first wash was saved for cytokine analyses (see below). Immune cells contained in the BAL fluid were collected by pooling the pellets from each wash. Cells were resuspended in fluorescence-activated cell sorting (FACS) buffer ( $2 \%$ foetal bovine serum in PBS). A $50-\mu l$ aliquot was taken, the total number of cells in BAL fluid were counted on a hemocytometer, and viability was determined by Typran Blue exclusion. Before antibody staining, cells were incubated with Fixable Viability Stain 450 (BD Biosciences) for $15 \mathrm{~min}$ at room temperature. Cells were then washed with FACS buffer $+0.1 \%$ sodium azide and incubated with anti-CD16/32 FcBlock antibody (eBioscience, clone 93) to minimize non-specific binding for $10 \mathrm{~min}$ at $4^{\circ} \mathrm{C}$. For ex vivo cell surface marker detection, cells were stained with antibodies for CD45 (BD Biosciences, clone 30-F11), CD11b (BD Biosciences, clone M1/70), F4/80 (eBioscience, clone BM8), CD11c (BD Biosciences, clone HL3) and Ly6G (BD Biosciences, clone 1A8). All antibodies were used at 1:100 dilutions in FACS buffer, while Live/Dead cell stain was used at 1:1,000. Cells were washed with FACS buffer and fixed with $2 \%$ paraformaldehyde. Before analysis, samples were plated for sterility onto BHI.
Samples were analysed using a BD FACSCanto II flow cytometer and FlowJo software. Statistical significance was calculated by the Student's $t$-test.

Cytokine analysis. At $48 \mathrm{~h}$ post inoculation with PBS or the indicated $Y$. pestis strains, the levels of tumour-necrosis factor, interferon- $\gamma$, monocyte chemoattractant protein 1 (MCP-1), and interleukin-6 were quantitatively established from the supernatants of collected BAL fluid (see above) using the cytometric bead array technique (BD Cytometric Bead Array Mouse Inflammation Kit, BD Biosciences) as specified by the manufacture ${ }^{38}$. Before analysis, supernatants were passed through a $0.22-\mu \mathrm{m}$ filter and plated for sterilization. Data were analysed using the BD Cytometric Bead Array Software. Statistical significance was calculated by the Student's $t$-test.

Statistics. In all cases, statistical means are graphed and error bars represent s.e.m. Sample sizes $(n)$ given represent biological replicates and were not predetermined. For animal experiments, sample sizes were limited by vivarium and ABSL-3 facility capabilities and experimental processing times. Any variation in animal age was matched between groups of interest for comparison and animals were euthanized for processing in the same order as infection. Within a given group, the orders of animal infection and euthanasia were random. The blinding of animal experimental groups was not possible. Student's two-tailed unpaired $t$-test was performed for all instances, unless otherwise indicated. All experiments were analysed with the statistical tests indicated using GraphPad Prism 5. $P$ values are indicated as ${ }^{*} P \leq 0.05,{ }^{*} P \leq 0.01$ and ${ }^{* *} P \leq 0.001$.

\section{References}

1. Perry, R. D. \& Fetherston, J. D. Yersinia pestis-etiologic agent of plague. Clin. Microbiol. Rev. 10, 35-66 (1997).

2. Wren, B. W. The yersiniae-a model genus to study the rapid evolution of bacterial pathogens. Nat. Rev. Microbiol. 1, 55-64 (2003).

3. Achtman, M. et al. Microevolution and history of the plague bacillus, Yersinia pestis. Proc. Natl Acad. Sci. USA 101, 17837-17842 (2004).

4. Achtman, M. et al. Yersinia pestis, the cause of plague, is a recently emerged clone of Yersinia pseudotuberculosis. Proc. Natl Acad. Sci. USA 96, 14043-14048 (1999).

5. Morelli, G. et al. Yersinia pestis genome sequencing identifies patterns of global phylogenetic diversity. Nat. Genet. 42, 1140-1143 (2010).

6. Cui, Y. et al. Historical variations in mutation rate in an epidemic pathogen, Yersinia pestis. Proc. Natl Acad. Sci. USA 110, 577-582 (2013).

7. Sun, Y. C., Jarrett, C. O., Bosio, C. F. \& Hinnebusch, B. J. Retracing the evolutionary path that led to flea-borne transmission of Yersinia pestis. Cell Host Microbe 15, 578-586 (2014).

8. Chain, P. S. et al. Insights into the evolution of Yersinia pestis through whole-genome comparison with Yersinia pseudotuberculosis. Proc. Natl Acad. Sci. USA 101, 13826-13831 (2004).

9. Derbise, A. et al. Delineation and analysis of chromosomal regions specifying Yersinia pestis. Infect Immun. 78, 3930-3941 (2010).

10. Pouillot, F., Fayolle, C. \& Carniel, E. Characterization of chromosomal regions conserved in Yersinia pseudotuberculosis and lost by Yersinia pestis. Infect Immun. 76, 4592-4599 (2008).

11. Chouikha, I. \& Hinnebusch, B. J. Silencing urease: A key evolutionary step that facilitated the adaptation of Yersinia pestis to the flea-borne transmission route. Proc. Natl Acad. Sci. USA 111, 18709-18714 (2014).

12. Reuter, S. et al. Parallel independent evolution of pathogenicity within the genus Yersinia. Proc. Natl Acad. Sci. USA 111, 6768-6773 (2014).

13. Skurnik, M., Peippo, A. \& Ervela, E. Characterization of the O-antigen gene clusters of Yersinia pseudotuberculosis and the cryptic O-antigen gene cluster of Yersinia pestis shows that the plague bacillus is most closely related to and has evolved from Y. pseudotuberculosis serotype O:1b. Mol. Microbiol. 37, 316-330 (2000).

14. Forman, S. et al. yadBC of Yersinia pestis, a new virulence determinant for bubonic plague. Infect Immun. 76, 578-587 (2008).

15. Maldonado-Arocho, F. J., Green, C., Fisher, M. L., Paczosa, M. K. \& Mecsas, J. Adhesins and host serum factors drive Yop translocation by Yersinia into professional phagocytes during animal infection. PLoS Pathog. 9, e1003415 (2013).

16. Heise, T. \& Dersch, P. Identification of a domain in Yersinia virulence factor YadA that is crucial for extracellular matrix-specific cell adhesion and uptake. Proc. Natl Acad. Sci. USA 103, 3375-3380 (2006).

17. Sebbane, F., Jarrett, C. O., Gardner, D., Long, D. \& Hinnebusch, B. J. Role of the Yersinia pestis plasminogen activator in the incidence of distinct septicemic and bubonic forms of flea-borne plague. Proc. Natl Acad. Sci. USA 103, 5526-5530 (2006).

18. Lathem, W. W., Crosby, S. D., Miller, V. L. \& Goldman, W. E. Progression of primary pneumonic plague: a mouse model of infection, pathology, and bacterial transcriptional activity. Proc. Natl Acad. Sci. USA 102, 17786-17791 (2005). 
19. Caulfield, A. J. \& Lathem, W. W. Substrates of the plasminogen activator protease of Yersinia pestis. Adv. Exp. Med. Biol. 954, 253-260 (2012).

20. Suomalainen, M. et al. Using every trick in the book: the Pla surface protease of Yersinia pestis. Adv. Exp. Med. Biol. 603, 268-278 (2007).

21. Zhang, S. S. et al. Plasminogen activator Pla of Yersinia pestis utilizes murine DEC-205 (CD205) as a receptor to promote dissemination. J. Biol. Chem. 283, 31511-31521 (2008)

22. Degen, J. L., Bugge, T. H. \& Goguen, J. D. Fibrin and fibrinolysis in infection and host defense. J. Thromb. Haemost. 5(Suppl 1): 24-31 (2007).

23. Kukkonen, M. et al. Protein regions important for plasminogen activation and inactivation of alpha2-antiplasmin in the surface protease Pla of Yersinia pestis. Mol. Microbiol. 40, 1097-1111 (2001).

24. Kukkonen, M. et al. Lack of $\mathrm{O}$-antigen is essential for plasminogen activation by Yersinia pestis and Salmonella enterica. Mol. Microbiol. 51, 215-225 (2004).

25. Korhonen, T. K., Haiko, J., Laakkonen, L., Jarvinen, H. M. \& Westerlund-Wikstrom, B. Fibrinolytic and coagulative activities of Yersinia pestis. Front. Cell. Infect. Microbiol. 3, 35 (2013).

26. Song, Y. et al. Complete genome sequence of Yersinia pestis strain 91001, an isolate avirulent to humans. DNA Res. 11, 179-197 (2004).

27. Eppinger, M. et al. Genome sequence of the deep-rooted Yersinia pestis strain Angola reveals new insights into the evolution and pangenome of the plague bacterium. J. Bacteriol. 192, 1685-1699 (2010).

28. Haiko, J., Kukkonen, M., Ravantti, J. J., Westerlund-Wikstrom, B. \& Korhonen, T. K. The single substitution I259T, conserved in the plasminogen activator Pla of pandemic Yersinia pestis branches, enhances fibrinolytic activity. J. Bacteriol. 191, 4758-4766 (2009).

29. Filippov, A. A., Solodovnikov, N. S., Kookleva, L. M. \& Protsenko, O. A. Plasmid content in Yersinia pestis strains of different origin. FEMS Microbiol Lett. 55, 45-48 (1990).

30. Worsham, P. L. \& Roy, C. Pestoides F, a Yersinia pestis strain lacking plasminogen activator, is virulent by the aerosol route. Adv. Exp. Med. Biol. 529, 129-131 (2003).

31. Anisimov, A. P., Lindler, L. E. \& Pier, G. B. Intraspecific diversity of Yersinia pestis. Clin. Microbiol. Rev. 17, 434-464 (2004).

32. Rajanna, C. et al. Characterization of pPCP1 plasmids in Yersinia pestis strains isolated from the former Soviet Union. Int. J. Microbiol. 2010, 760819 (2010).

33. Wagner, D. M. et al. Yersinia pestis and the plague of Justinian 541-543 AD: a genomic analysis. Lancet Infect. Dis. 14, 319-326 (2014).

34. Schuenemann, V. J. et al. Targeted enrichment of ancient pathogens yielding the pPCP1 plasmid of Yersinia pestis from victims of the Black Death. Proc. Natl Acad. Sci. USA 108, E746-E752 (2011).

35. Garcia, E. et al. Pestoides F, an atypical Yersinia pestis strain from the former Soviet Union. Adv. Exp. Med. Biol. 603, 17-22 (2007).

36. Bearden, S. W. \& Brubaker, R. R. Recent findings regarding maintenance of enzootic variants of Yersinia pestis in sylvatic reservoirs and their significance in the evolution of epidemic plague. Vector Borne Zoonotic Dis. 10, 85-92 (2010).

37. Lathem, W. W., Price, P. A., Miller, V. L. \& Goldman, W. E. A plasminogen-activating protease specifically controls the development of primary pneumonic plague. Science 315, 509-513 (2007).

38. Caulfield, A. J., Walker, M. E., Gielda, L. M. \& Lathem, W. W. The Pla protease of Yersinia pestis degrades fas ligand to manipulate host cell death and inflammation. Cell Host Microbe 15, 424-434 (2014).

39. Brubaker, R. R. The recent emergence of plague: a process of felonious evolution. Microbial. Ecol. 47, 293-299 (2004).

40. Lawrence, J. G. Common themes in the genome strategies of pathogens. Curr. Opin. Genet. Dev. 15, 584-588 (2005).

41. Schrimpe-Rutledge, A. C. et al. Comparative omics-driven genome annotation refinement: application across Yersiniae. PLoS ONE 7, e33903 (2012).

42. Fisher, M. L., Castillo, C. \& Mecsas, J. Intranasal inoculation of mice with Yersinia pseudotuberculosis causes a lethal lung infection that is dependent on Yersinia outer proteins and PhoP. Infect Immun. 75, 429-442 (2007).

43. Kutyrev, V. et al. Expression of the plague plasminogen activator in Yersinia pseudotuberculosis and Escherichia coli. Infect Immun. 67, 1359-1367 (1999).

44. Pouillot, F. et al. Evaluation of O-antigen inactivation on Pla activity and virulence of Yersinia pseudotuberculosis harbouring the pPla plasmid. Microbiology 151, 3759-3768 (2005).

45. Sodeinde, O. A. et al. A surface protease and the invasive character of plague. Science 258, 1004-1007 (1992).

46. Welkos, S. L., Friedlander, A. M. \& Davis, K. J. Studies on the role of plasminogen activator in systemic infection by virulent Yersinia pestis strain C092. Microb Pathog. 23, 211-223 (1997).

47. Bearden, S. W. et al. Attenuated enzootic (pestoides) isolates of Yersinia pestis express active aspartase. Microbiology 155, 198-209 (2009).

48. Moran, N. A. Microbial minimalism: genome reduction in bacterial pathogens. Cell 108, 583-586 (2002).
49. Lorange, E. A., Race, B. L., Sebbane, F. \& Hinnebusch, B. J. Poor vector competence of fleas and the evolution of hypervirulence in Yersinia pestis. J. Infect. Dis. 191, 1907-1912 (2005).

50. Sun, Y. C., Hinnebusch, B. J. \& Darby, C. Experimental evidence for negative selection in the evolution of a Yersinia pestis pseudogene. Proc. Natl Acad. Sci. USA 105, 8097-8101 (2008).

51. Bliven, K. A. \& Maurelli, A. T. Antivirulence genes: insights into pathogen evolution through gene loss. Infect Immun. 80, 4061-4070 (2012).

52. Mandel, M. J., Wollenberg, M. S., Stabb, E. V., Visick, K. L. \& Ruby, E. G. A single regulatory gene is sufficient to alter bacterial host range. Nature 458, 215-218 (2009).

53. Damkiaer, S., Yang, L., Molin, S. \& Jelsbak, L. Evolutionary remodeling of global regulatory networks during long-term bacterial adaptation to human hosts. Proc. Natl Acad. Sci. USA 110, 7766-7771 (2013).

54. Viana, D. et al. A single natural nucleotide mutation alters bacterial pathogen host tropism. Nat. Genet. 47, 361-366 (2015).

55. Cathelyn, J. S., Crosby, S. D., Lathem, W. W., Goldman, W. E. \& Miller, V. L. RovA, a global regulator of Yersinia pestis, specifically required for bubonic plague. Proc. Natl Acad. Sci. USA 103, 13514-13519 (2006).

56. Perry, R. D. \& Bearden, S. W. Isolation and confirmation of Yersinia pestis mutants exempt from select agent regulations. Curr. Protoc Microbiol Chapter 5, Unit 5B.2 (2008).

57. Kado, C. I. \& Liu, S. T. Rapid procedure for detection and isolation of large and small plasmids. J. Bacteriol. 145, 1365-1373 (1981).

58. Bellows, L. E., Koestler, B. J., Karaba, S. M., Waters, C. M. \& Lathem, W. W. Hfq-dependent, co-ordinate control of cyclic diguanylate synthesis and catabolism in the plague pathogen Yersinia pestis. Mol. Microbiol. 86, 661-674 (2012).

59. Eddy, J. L., Gielda, L. M., Caulfield, A. J., Rangel, S. M. \& Lathem, W. W. Production of outer membrane vesicles by the plague pathogen Yersinia pestis. PLoS ONE 9, e107002 (2014).

60. Lathem, W. W. et al. Posttranscriptional regulation of the Yersinia pestis cyclic AMP receptor protein Crp and impact on virulence. mBio 5, e01038-01013 (2014).

61. Schiano, C. A. et al. Genome-wide analysis of small RNAs expressed by Yersinia pestis identifies a regulator of the Yop-Ysc type III secretion system. J. Bacteriol. 196, 1659-1670 (2014).

62. Choi, K. H. et al. A Tn7-based broad-range bacterial cloning and expression system. Nat. Methods 2, 443-448 (2005).

\section{Acknowledgements}

This work was supported by funding from Public Health Grants R01 AI093727 and R21 AI103658 to W.W.L. D.L.Z. was supported by funding from T32 AI007476. We thank Jeremy Ritzert, Lauren Bellows, Danielle O’Neal and Dr Adam Caulfield for technical assistance, and Dr Paul Keim for helpful discussions. We are grateful to Dr Susan Straley for providing the pPCP1::kan plasmid, Dr Robert Perry for providing the pCD1Ap plasmid, Y. pestis Angola, Pestoides A and E strains, and Dr Scott Bearden for providing the Y. pestis Pestoides F strain. This work was supported by the Northwestern University Interdepartmental Immunobiology Flow Cytometry Core Facility. Histopathology was performed by the Northwestern University Mouse Histology and Phenotyping Laboratory, supported by NCI CA060553. Imaging was performed at the Northwestern University Cell Imaging Facility, supported by NCI CCSG P30 CA060553 awarded to the Robert H. Lurie Comprehensive Cancer Center.

\section{Author contributions}

D.L.Z. and W.W.L. conceived and designed the experiments. D.L.Z., J.A.S., J.L.E. and W.W.L. performed the experiments. D.L.Z., J.A.S., J.L.E. and W.W.L. analysed the data. D.L.Z. and W.W.L wrote the manuscript.

\section{Additional information}

Supplementary Information accompanies this paper at http://www.nature.com/ naturecommunications

Competing financial interests: The authors declare no competing financial interests.

Reprints and permission information is available online at http://npg.nature.com/ reprintsandpermissions/

How to cite this article: Zimbler, D. L. et al. Early emergence of Yersinia pestis as a severe respiratory pathogen. Nat. Commun. 6:7487 doi: 10.1038/ncomms8487 (2015).

This work is licensed under a Creative Commons Attribution 4.0 International License. The images or other third party material in this article are included in the article's Creative Commons license, unless indicated otherwise in the credit line; if the material is not included under the Creative Commons license, users will need to obtain permission from the license holder to reproduce the material. To view a copy of this license, visit http://creativecommons.org/licenses/by/4.0/ 